\title{
COMPOSITION ALGEBRAS OVER ALGEBRAIC CURVES OF GENUS ZERO
}

\author{
HOLGER P. PETERSSON
}

Max Koecher in dankbarer Erinnerung gewidmet

\begin{abstract}
We rephrase the classical theory of composition algebras over fields, particularly the Cayley-Dickson Doubling Process and Zorn's Vector Matrices, in the setting of locally ringed spaces. Fixing an arbitrary base field, we use these constructions to classify composition algebras over (complete smooth) curves of genus zero. Applications are given to composition algebras over function fields of genus zero and polynomial rings.
\end{abstract}

\section{INTRODUCTION}

Our main concern in this paper is to initiate the study of nonassociative algebras over algebraic varieties. As we shall see below, even ordinary finitedimensional algebras over fields may benefit from such an investigation. Rather than treating the subject in excessive generality, we prefer to focus attention on the classification problem for the most elementary among the interesting classes of algebras (composition algebras) over the most elementary among the interesting classes of varieties (curves of genus zero); the base field is arbitrary.

Along the way towards classification, we find it convenient to rephrase the theory of composition algebras in the language of locally ringed spaces, leading, after some terminological preliminaries in $\S 1$, to relative versions of the Cayley-Dickson-Doubling Process (2.5) and Zorn's Vector Matrices (3.5); the former is related to the concept of a quadratic space relative to a "Hamiltonian" quaternion algebra introduced by Knus [Kn]. The proof of the Classification Theorem (4.4) will be carried out in $\S 5$. The critical step is to show that a composition algebra of rank at least two over a curve $X$ of genus zero contains a composition subalgebra of rank exactly two defined over the base field (5.5). The Classification Theorem then follows by the method of descent. As a byproduct of the proof one obtains the additional result (4.5) that, roughly speaking, composition algebras of rank $r$ over $X$ containing a nonsplit composition subalgebra of rank $\frac{r}{2}$ which is defined over the base field are, with one exception, themselves defined over the base field.

Due to the intimate relationship between octonion algebras and groups of type $G_{2}$, the present investigation may be regarded as a supplement, carried

Received by the editors April 13, 1990 and, in revised form, February 26, 1991.

1980 Mathematics Subject Classification (1985 Revision). Primary 17A75, 14H99; Secondary 14F05, 17A45, 17D05. 
out on an elementary level, of Harder's fundamental work on algebraic group schemes over complete curves. As Harder himself has pointed out [H, pp. 147148], one should be able, at least in principle, to classify the semisimple ones among these objects once the underlying curve has genus zero, and it is quite conceivable that such a classification more or less directly implies our own Classification Theorem 4.4. Even if this should be the case, however, the approach adopted here seems to deserve some independent interest since it rather quickly leads to results that are quite explicit. Moreover, it immediately suggests generalizations to curves of higher genus as well as to more general classes of algebras.

Two applications of our results to finite-dimensional nonassociative algebras over fields will be discussed in this paper. In 3.8 we construct a whole series of quadratic alternative algebras with big radicals which do not square to zero by considering split octonions over projective $n$-space and passing to global sections. In $\S 6$ we concern ourselves with composition algebras over function fields of genus zero and polynomial rings. Guided by a classical theorem of Harder on symmetric bilinear forms over polynomial rings, we show in particular (6.8) that an octonion algebra over the polynomial ring $k[t]$ is defined over $k$, for any field $k$ which is perfect or of characteristic not two.

The terminology adopted in this paper is the standard one; concepts from algebraic geometry not explained in the text are to be understood in the sense of Hartshorne [Ha]. The author is greatly indebted to R. Börger, H. Lindel, G. Schabhüser, W. Scharlau, M. Schulte, M. Slater and, in particular, to O. Loos for useful conversations on the subject; also, to M. Knus for having supplied him with a copy of $[\mathrm{Kn}]$ as well as with references concerning the Brauer groups of the affine and projective line. Finally, the extremely useful comments by the referee, having improved considerably upon the original version of the paper, are gratefully acknowledged. Special cases of our results have been announced in [P2].

\section{Composition Algebras OVER LOCALly RINGED SPACES}

1.1. Let $R$ be a (unital commutative associative) ring of scalars. An $R$-module $M$ is said to have full support if $\operatorname{Supp} M=\operatorname{Spec} R$, so $M_{\mathfrak{p}} \neq 0$ for all prime ideals $\mathfrak{p} \subset R$. Given a quadratic form $q: M \rightarrow R$, the bilinear form induced by $q$ will be written as

$$
M \times M \rightarrow R,(u, v) \mapsto q(u, v)=q(u+v)-q(u)-q(v) .
$$

The term “ $R$-algebra" always refers to unital nonassociative algebras over $R$ which are finitely generated projective as $R$-modules. An $R$-algebra $C$ is said to be quadratic in case there exists a quadratic form $n: C \rightarrow R$ satisfying the following two conditions:

QA1. $n$ is unital, so $n\left(1_{C}\right)=1$.

QA2. Each $u \in C$ satisfies the equation

$$
u^{2}-t(u) u+n(u) 1_{C}=0 \quad\left(t(u)=n\left(1_{C}, u\right)\right) .
$$

Quadratic algebras are invariant under base change.

1.2 Lemma. Let $C$ be a quadratic algebra over $R$. Then there exists a unique quadratic form $n$ on $C$ satisfying conditions QA1, 2 above.

Proof. ${ }^{1}$ By standard facts about localizations, the statement is local on $R$, so

\footnotetext{
${ }^{1}$ This proof grew out of a conversation with G. Schabhüser.
} 
we may assume that $R$ is a local ring, with maximal ideal $\mathfrak{m}$, and hence $C$ is free as an $R$-module. Since $n$ is unital, we may extend $1_{C} \notin \mathfrak{m} C$ to a basis of $C$, and the assertion follows from [M, 2.3(vi)]. Q.E.D.

1.3. Because of 1.2 , we are now allowed to call $n=n_{C}$ the norm, $t=t_{C}$, defined by $t(u)=n\left(1_{C}, u\right)$, the trace, and

$$
{ }^{*}: C \rightarrow C, u \mapsto u^{*}=t(u) 1_{C}-u
$$

the canonical reflection of the quadratic algebra $C$ over $R$.

1.4. An $R$-algebra $C$ is said to be a composition algebra in case it has full support (1.1) and there exists a quadratic form $n: C \rightarrow R$ satisfying the following two conditions:

CA1. The symmetric bilinear form induced by $n$ is nondegenerate, so determines a module isomorphism $C \stackrel{\sim}{\rightarrow} \check{C}=\operatorname{Hom}_{R}(C, R)$.

CA2. $n$ permits composition, so $n(u v)=n(u) n(v)$ for all $u, v \in C$.

Since a composition algebra $C$ over $R$ by definition has full support, hence must be unitally faithfull in the sense of [M, p. 85], it follows from [M, 4.6] that $C$ is alternative (so the associator $(u v) w-u(v w)$ is alternating) as well as quadratic in the sense of 1.1 ; more precisely, the quadratic form $n$ on $C$ satisfying conditions CA1, 2 above is unique and, in fact, agrees with the norm of $C$ in the sense of 1.3. Also, the canonical reflection of $C$ is an algebra involution [M, p. 96], called the canonical involution of $C$. Finally, composition algebras are invariant under base change.

1.5. Now fix a locally ringed space $X$, with structure sheaf $\mathscr{O}_{X}$. For $P \in X$, we denote by $\mathscr{O}_{P, X}$ (or simply $\mathscr{O}_{P}$ if no confusion can arise) the local ring of $\mathscr{O}_{X}$ at $P$, by $\mathfrak{m}_{P}=\mathfrak{m}_{P, X}$ the maximal ideal of $\mathscr{O}_{P}$, and by $\boldsymbol{\kappa}(P)=\mathscr{O}_{P} / \mathfrak{m}_{P}$ the corresponding residue class field; the stalk of an $\mathscr{O}_{X}$-module $\mathscr{F}$ at $P$ will be denoted by $\mathscr{F}_{P}$. As in $1.1, \mathscr{F}$ is said to have full support if $\operatorname{Supp} \mathscr{F}=X$, so $\mathscr{F}_{P} \neq 0$ for all $P \in X$. An $\mathscr{O}_{X}$-module $\mathscr{E}$ is said to be locally free of finite rank if each $P \in X$ admits an open neighborhood $P \in U \subset X$ such that $\left.\mathscr{E}\right|_{U} \cong \mathscr{O}_{U}^{n}$, for some integer $n \geq 0$ (which may depend on $P$ ). We then define the rank of $\mathscr{E}$ as $\sup \left\{\operatorname{rank}_{\mathscr{O}_{P}} \mathscr{E}_{P} ; P \in X\right\}$ (which is either an integer or $+\infty$ ). (Nonassociative) algebras over $X$ are always tacitly assumed to be unital and locally free of finite rank as $\mathscr{O}_{X}$-modules.

1.6. As in 1.4 we define a composition algebra over $X$ to be an $\mathscr{O}_{X}$-algebra $\mathscr{C}$ which has full support and admits a quadratic form $N: \mathscr{C} \rightarrow \mathscr{O}_{X}$ satisfying the following conditions:

$\mathscr{C} \mathscr{A} 1$. The symmetric bilinear form induced by $N$ is nondegenerate, so determines a module isomorphism $\mathscr{C} \stackrel{\sim}{\rightarrow} \check{\mathscr{C}}=\mathscr{H}_{m_{X}}\left(\mathscr{C}, \mathscr{O}_{X}\right)$.

$\mathscr{C} \mathscr{A}$ 2. $N$ permits composition, so $N(u v)=N(u) N(v)$ for all sections $u$, $v$ of $\mathscr{C}$ over the same open subset of $X$.

Quadratic (resp. alternative) algebras over $X$ are defined in a similar manner, and it is clear that 1.2-1.4 carry over to this more general setting. In particular, we may talk about the norm, trace, canonical involution of a composition algebra $\mathscr{C}$ over $X$, denoted by $N=N_{\mathscr{C}}, T=T_{\mathscr{C}},{ }^{*}$, respectively; also, we have the following result. 
1.7 Proposition. (a) Let $\mathscr{C}$ be an algebra over $X$ and $N: \mathscr{C} \rightarrow \mathscr{O}_{X}$ a quadratic form. Then $\mathscr{C}$ is a composition algebra over $X$ with norm $N$ if and only if, for each $P \in X, \mathscr{C}_{P}$ is a composition algebra over $\mathscr{O}_{P}$ with norm $N_{P}$.

(b) Composition algebras are invariant under base change: If $\sigma: X^{\prime} \rightarrow X$ is a morphism of locally ringed spaces, and $\mathscr{C}$ is a composition algebra over $X$, $\sigma^{*} \mathscr{C}=\mathscr{C} \otimes_{\mathscr{O}_{X}} \mathscr{O}_{X^{\prime}}$ is a composition algebra over $X^{\prime}$.

(c) Composition algebras are quadratic alternative.

(d) Composition algebras exist only in ranks 1, 2, 4, 8.

(e) A composition algebra is associative if and only if it has rank at most 4; it is commutative associative if and only if it has rank at most 2 .

Proof. Since composition algebras by definition have full support, (a) is obvious. (b)-(d) follow immediately from the corresponding statements in 1.4 and standard properties of composition algebras over fields by passing to the stalks $\mathscr{C}_{P}$ and to the residue class algebras $\mathscr{C}_{P} \otimes_{\mathscr{O}_{P}} \boldsymbol{\kappa}(P)$, for $P \in X$. (e) By Nakayama's Lemma, the property of a composition algebra over a field to be commutative associative (resp. associative) if and only if it has rank at most 2 (resp. 4) if and only if it may be generated by one (resp. two) elements(s) carries over to composition algebras over local rings. Hence (e) follows from (a). Q.E.D.

1.8. A composition algebra over $X$ is said to be a torus (resp. a quaternion algebra, resp. an octonion algebra) in case it has constant rank 2 (resp. 4, resp. 8). For example,

$$
\mathscr{O}_{X} \oplus \mathscr{O}_{X} \quad \text { (direct sum of ideals) }
$$

is a torus, its norm being given by the hyperbolic quadratic form $(a, b) \mapsto a b$. A composition algebra over $X$ is said to be split if it contains an isomorphic copy of $\mathscr{O}_{X} \oplus \mathscr{O}_{X}$ as a composition subalgebra.

1.7 (c) implies that, for any open subset $U$ of $X, \Gamma(U, \mathscr{C})$ (i.e., the set of sections of the sheaf $\mathscr{C}$ over $U$ ) is a quadratic alternative algebra over $\Gamma\left(U, \mathscr{O}_{X}\right)$, without, however, being in general a composition algebra. In fact, examples will be specified below where this algebra has a highly degenerate norm form and, consequently, a big radical. 1.7(d) has been obtained earlier in a slightly different context by Legrand [Le].

1.9. Let $Y=\operatorname{Spec} R$ be an affine scheme. Under the usual categorical equivalence, (composition) algebras over $Y$ in the sense of 1.6 are basically the same as (composition) algebras over $R$ in the sense of 1.4. We will not always distinguish carefully between these two notions. If $X$ is an $R$-scheme, with structure morphism $\tau: X \rightarrow Y$, a (composition) algebra $\mathscr{C}$ over $X$ is said to be defined over $R$ in case there exists a (composition) algebra $C$ over $R$ such that $\mathscr{C} \cong \tau^{*} C$. We close this section by giving an elementary but useful criterion for a composition algebra to be defined over the base ring.

1.10 Lemma. Let $X$ be a scheme over the affine scheme $Y=\operatorname{Spec} R$ and suppose $\Gamma\left(X, \mathscr{O}_{X}\right)=R$. Then a (composition) algebra $\mathscr{C}$ over $X$ is defined over $R$ providing it is globally free as an $\mathscr{O}_{X}$-module.

Proof. With $n=\operatorname{rank} \mathscr{C}, R^{n}=\Gamma\left(X, \mathscr{O}_{X}^{n}\right) \cong \Gamma(X, \mathscr{C})$ carries the structure of a (composition) algebra over $R$ whose base change to $X$ becomes canonically isomorphic to $\mathscr{C}$. Q.E.D. 


\section{The Cayley-Dickson Doubling Process}

2.1. Let $D$ be an associative composition algebra over the ring $R$ and $\mu \in R$ be a unit. Then Albert [A] has shown that the $R$-module $D \oplus D$ becomes a composition algebra under the multiplication

$$
(u, v)\left(u^{\prime}, v^{\prime}\right)=\left(u u^{\prime}+\mu v^{\prime *} v, v^{\prime} u+v u^{\prime *}\right)
$$

for $u, v, u^{\prime}, v^{\prime} \in D$. This composition algebra, denoted by $\operatorname{Cay}(D, \mu)$, is traditionally said to arise from $D, \mu$ by means of the Cayley-Dickson Doubling Process. Its norm relates to the norm of $D$ via the formula

$$
n_{\text {Cay }(D, \mu)}((u, v))=n_{D}(u)-\mu n_{D}(v) .
$$

The imbedding of $D$ to the first summand of $\operatorname{Cay}(D, \mu)$ is an algebra monomorphism. If $D$ has rank at most two, $\operatorname{Cay}(D, \mu)$ is associative, and we abbreviate the iterated Cayley-Dickson construction $\operatorname{Cay}(\operatorname{Cay}(D, \mu), \nu)$ by $\operatorname{Cay}(D ; \mu, \nu)$.

2.2 Lemma. Let $D$ be a proper composition subalgebra of a quadratic alternative algebra $C$ over $R$.

(a) Suppose $l \in D^{\perp}$, the orthogonal complement of $D$ in $C$ relative to $n_{C}$, is invertible in $C$, and put $\mu=-n_{C}(l)$. Then the imbedding $D \hookrightarrow C$ uniquely extends to an imbedding $\operatorname{Cay}(D, \mu) \hookrightarrow C$ sending $\left(0,1_{D}\right)$ to $l$.

(b) If $C$ is a composition algebra and $R$ is a local ring, there always exists an $l \in D^{\perp}$ which is invertible in $C$.

Proof. (a) is the fundamental fact underlying the structure theory of composition algebras over fields. In this generality, it is due to McCrimmon [M, 6.5].

(b) is well known and trivial for composition algebras over fields. In the general situation, we pass to the base change $C^{\prime}=C \otimes_{R} k$ from $R$ to its residue class field and find an $l^{\prime} \in\left(D \otimes_{R} k\right)^{\perp}=D^{\perp} \otimes_{R} k$ which is invertible in $C^{\prime}$. Lifting $l^{\prime}$ to $D^{\perp}$ now yields an element $l$ of the desired kind. Q.E.D.

2.3. Given an algebra $\mathscr{H}$ of "Hamiltonian quaternions" over a real scheme, Knus [Kn, 1.3] has introduced the notion of a quadratic space of type $\mathscr{H}$ and has constructed universal gadgets in this context [Kn, 1.5, 1.6]. For our purpose it will be convenient to rephrase this part of his work in the more general setting of locally ringed spaces.

So let $X$ be a locally ringed space, $\mathscr{D}$ an associative composition algebra over $X$ and $\mathscr{P}$ a locally free right $\mathscr{D}$-module of (constant) rank one. We write $\mathscr{D}^{\times}$for the sheaf of units of $\mathscr{D}$. Matching canonically the pointed set of isomorphism classes of locally free right $\mathscr{D}$-modules of rank one with $\check{H}^{1}\left(X, \mathscr{D}^{\times}\right)$in the sense of noncommutative Čech cohomology [Mi, III, 4.6], and noting that the morphism $N_{\mathscr{D}}: \mathscr{D}^{\times} \rightarrow \mathscr{O}_{X}^{\times}$of group sheaves determines a morphism

$$
N_{\mathscr{D}}: \check{H}^{1}\left(X, \mathscr{D}^{\times}\right) \rightarrow \check{H}^{1}\left(X, \mathscr{O}_{X}^{\times}\right)=\operatorname{Pic} X
$$

of pointed sets, $N_{\mathscr{D}}(\mathscr{P})$ is an invertible sheaf on $X$, called the norm of $\mathscr{P}$. We say $\mathscr{P}$ is of norm one if $N_{\mathscr{D}}(\mathscr{P}) \cong \mathscr{O}_{X}$. A quadratic map $Q: \mathscr{P} \rightarrow \mathscr{F}$ in the category of $\mathscr{O}_{X}$-modules is said to be multiplicative in case

$$
Q(w \cdot u)=N_{\mathscr{D}}(u) Q(w)
$$

for all sections $w$ in $\mathscr{P}, u$ in $\mathscr{D}$, the dot on the left referring to the right $\mathscr{D}$ module structure of $\mathscr{P}$. By a norm on $\mathscr{P}$ we mean a multiplicative quadratic 
map $N: \mathscr{P} \rightarrow N_{\mathscr{D}}(\mathscr{P})$ whose induced symmetric bilinear map is nondegenerate, i.e., induces a linear isomorphism $\mathscr{P} \stackrel{\sim}{\rightarrow} \operatorname{Hom}_{X}\left(\mathscr{P}, N_{\mathscr{D}}(\mathscr{P})\right)$.

2.4. Keeping the notations of 2.3 , norms on $\mathscr{P}$ always exist and are unique up to an invertible factor in $\Gamma\left(X, \mathscr{O}_{X}\right)$. Indeed, let $\mathfrak{U}=\left(U_{i}\right)_{i \in I}$ be an open cover of $X$ such that the right $\mathscr{D}$-module $\mathscr{P}$ becomes free of rank one over each $U_{i}$, with basis vector $l_{i} \in \Gamma\left(U_{i}, \mathscr{P}\right)$. Then $l_{j}=l_{i} \cdot u_{i j}$ over $U_{i j}=U_{i} \cap U_{j}$, for some $u_{i j} \in \Gamma\left(U_{i j}, \mathscr{D}^{\times}\right)$, and $\alpha=\left(u_{i j}\right)$ is a cocycle of $\mathscr{D}^{\times}$over $X$ which determines $\mathscr{P}$ in $\breve{H}^{1}\left(X, \mathscr{D}^{\times}\right)$. Hence, setting $u_{i j}^{\prime}=N_{\mathscr{D}}\left(u_{i j}\right), \alpha^{\prime}=\left(u_{i j}^{\prime}\right)$ is a cocycle of $\mathscr{O}_{X}^{\times}$over $X$ which determines $\mathscr{L}=N_{\mathscr{D}}(\mathscr{P})$ in Pic $X$, so we may assume that $\mathscr{L}$ becomes free over each $U_{i}$, with basis vector $l_{i}^{\prime} \in \Gamma\left(U_{i}, \mathscr{L}\right)$ satisfying $l_{j}^{\prime}=l_{i}^{\prime} u_{i j}^{\prime}$ over $U_{i j}$. Now it follows easily that the multiplicative quadratic maps $N_{i}:\left.\left.\mathscr{P}\right|_{U_{i}} \rightarrow \mathscr{L}\right|_{U_{i}}$ sending $l_{i}$ to $l_{i}^{\prime}(i \in I)$ glue to give a norm $N$ on $\mathscr{P}$. Also, as observed in [Kn, 1.5], $N$ is universal (in the obvious sense) in the category of multiplicative quadratic maps, implying its uniqueness up to a factor in $\Gamma\left(X, \mathscr{O}_{X}^{\times}\right)$. Q.E.D.

2.5 Cayley-Dickson Doubling Theorem. (a) Let $\mathscr{D}$ be an associative composition algebra over the locally ringed space $X, \mathscr{P}$ a locally free right $\mathscr{D}$-module of rank one and norm one and $N: \mathscr{P} \rightarrow N_{\mathscr{D}}(\mathscr{P})=\mathscr{O}_{X}$ a norm on $\mathscr{P}$. Then there is a unique $\mathscr{O}_{X}$-bilinear map $\mathscr{P} \times \mathscr{P} \rightarrow \mathscr{D}$, written multiplicatively and satisfying

$$
(w \cdot u)(w \cdot v)=N(w) v^{*} u
$$

for all $u, v$ in $\mathscr{D}, w$ in $\mathscr{P}$. Also, the $\mathscr{O}_{X}$-module

$$
\operatorname{Cay}(\mathscr{D}, \mathscr{P}, N)=\mathscr{D} \oplus \mathscr{P}
$$

becomes a composition algebra under the multiplication

$$
(u, w)\left(u^{\prime}, w^{\prime}\right)=\left(u u^{\prime}+w w^{\prime}, w^{\prime} \cdot u+w \cdot u^{\prime *}\right),
$$

with norm $N_{\text {Cay }(\mathscr{D}, \mathscr{P}, N)}=N_{\mathscr{D}} \oplus(-N)$.

(b) Conversely, suppose $\mathscr{C}$ is a composition algebra of constant rank $r$ over $X$ and $\mathscr{D} \subset \mathscr{C}$ a composition subalgebra of constant rank $\frac{r}{2}$. Then there exist a locally free right $\mathscr{D}$-module $\mathscr{P}$ of rank one and norm one as well as a norm $N$ on $\mathscr{P}$ such that the identity of $\mathscr{D}$ extends to an isomorphism $\operatorname{Cay}(\mathscr{D}, \mathscr{P}, N) \stackrel{\sim}{\rightarrow}$ $\mathscr{C}$.

Proof. (a) $U_{i}, l_{i}, i \in I$, having the same meaning as in 2.4 , we set $\lambda_{i}=N\left(l_{i}\right) \in$ $\Gamma\left(U_{i}, \mathscr{O}_{X}\right)$ and obtain a bilinear map of the desired kind by glueing the local data $\left(\left.\mathscr{P}\right|_{U_{i}}\right) \times\left.\left(\left.\mathscr{P}\right|_{U_{i}}\right) \rightarrow \mathscr{D}\right|_{U_{i}}$ given by

$$
\left(l_{i} \cdot u\right)\left(l_{i} \cdot v\right)=\lambda_{i} v^{*} u
$$

for $u, v$ in $\left.\mathscr{D}\right|_{U_{i}}$. Uniqueness is clear locally, hence globally. That $\operatorname{Cay}(\mathscr{D}, \mathscr{P}, N)$ does indeed become a composition algebra over $X$ as indicated is now a matter of straightforward verification.

(b) We write $\mathscr{P}=\mathscr{D}^{\perp}$ for the orthogonal complement of $\mathscr{D}$ in $\mathscr{C}$ relative to $N_{\mathscr{C}}$. Then the action

$$
\mathscr{P} \times \mathscr{D} \rightarrow \mathscr{P},(w, u) \mapsto w \cdot u=u w,
$$

where the right-hand side refers to multiplication in $\mathscr{C}$, gives $\mathscr{P}$ the structure of a right $\mathscr{D}$-module which is locally free of rank one $(1.7(\mathrm{a}), 2.2)$. Choosing 
$\mathfrak{U}, l_{i}, u_{i j}$ as in 2.4 , and setting $\lambda_{i}=N_{\mathscr{C}}\left(l_{i}\right)$, we conclude $N_{\mathscr{D}}\left(u_{i j}\right)=\lambda_{i}^{-1} \lambda_{j}$ over $U_{i j}$. Hence $N_{\mathscr{D}}(\mathscr{P}) \cong \mathscr{O}_{X}$. Clearly, the restriction of $-N_{\mathscr{C}}$ to $\mathscr{D}^{\perp}$ makes up a norm $N$ on $\mathscr{P}$ and $\operatorname{Cay}(\mathscr{D}, \mathscr{P}, N)=\mathscr{C}$. Q.E.D.

2.6 Remark. The construction of $\operatorname{Cay}(\mathscr{D}, \mathscr{P}, N)$ is functorial in $\mathscr{P}, N$ : If $\varphi:(\mathscr{P}, N) \rightarrow\left(\mathscr{P}^{\prime}, N^{\prime}\right)$ is a morphism, i.e., a $\mathscr{D}$-linear map $\mathscr{P} \rightarrow \mathscr{P} \prime$ preserving norms,

$$
\mathbf{1}_{\mathscr{D}} \oplus \varphi: \operatorname{Cay}(\mathscr{D}, \mathscr{P}, N) \stackrel{\sim}{\rightarrow} \operatorname{Cay}\left(\mathscr{D}, \mathscr{P}^{\prime}, N^{\prime}\right)
$$

is an isomorphism of composition algebras. As a matter of terminology, the bilinear map $\mathscr{P} \times \mathscr{P} \rightarrow \mathscr{D}$ of $2.5(\mathrm{a})$ is said to be associated with $N$.

2.7 Corollary. Let $\mathscr{C}$ be a split quaternion algebra over $X$. Then there exists an invertible sheaf $\mathscr{L}$ on $X$ such that

$$
\mathscr{C} \cong \mathscr{E}_{n}{ }_{X}\left(\mathscr{L} \oplus \mathscr{O}_{X}\right)=\left(\begin{array}{cc}
\mathscr{O}_{X} & \mathscr{L} \\
\mathscr{L} & \mathscr{O}_{X}
\end{array}\right)
$$

the module on the right being equipped with ordinary matrix multiplication. More precisely, given a split torus $\mathscr{D} \subset \mathscr{C}$, there exists an isomorphism $\mathscr{C} \stackrel{\sim}{\rightarrow}$ End ${ }_{X}\left(\mathscr{L} \oplus \mathscr{O}_{X}\right)$ sending $\mathscr{D}$ to the diagonal of $\mathscr{E}_{\text {nd }}{ }_{X}\left(\mathscr{L} \oplus \mathscr{O}_{X}\right)$.

Proof. We may identify $\mathscr{D}$ with $\mathscr{O}_{X} \oplus \mathscr{O}_{X}(1.8)$ and have $\mathscr{C}=\operatorname{Cay}(\mathscr{D}, \mathscr{P}, N)$ as in 2.5. Since $\check{H}^{1}\left(X, \mathscr{D}^{\times}\right)=\operatorname{Pic} X \oplus \operatorname{Pic} X$, we have $\mathscr{P} \cong \mathscr{L} \oplus \mathscr{M}$, with invertible $\mathscr{O}_{X}$-modules $\mathscr{L}, \mathscr{M}$, forcing $\mathscr{L} \otimes \mathscr{M} \cong N_{\mathscr{D}}(\mathscr{P}) \cong \mathscr{O}_{X}$ and allowing us to assume $\mathscr{P}=\mathscr{L} \oplus \check{\mathscr{L}}$. By $2.4, N=\mu N^{0}$ for some $\mu \in \Gamma\left(X, \mathscr{O}_{X}^{\times}\right)$, where $N^{0}$ is the hyperbolic quadratic form on $\mathscr{L} \oplus \check{\mathscr{L}}$. The bilinear map $\mathscr{P} \times \mathscr{P} \rightarrow \mathscr{D}$ associated with $N(2.6)$ is then given by

$$
(s, \check{s})(t, \check{t})=\mu(\langle s, \check{t}\rangle,\langle t, \check{s}\rangle)
$$

for $s, t$ in $\mathscr{L}, \check{s}, \check{t}$ in $\check{\mathscr{L}},\langle$,$\rangle being the canonical pairing \mathscr{L} \times \check{\mathscr{L}} \rightarrow \mathscr{O}_{X}$. Now

defined by

$$
\varphi: \operatorname{Cay}(\mathscr{D}, \mathscr{P}, N) \rightarrow\left(\begin{array}{cc}
\mathscr{O}_{X} & \mathscr{L} \\
\mathscr{L} & \mathscr{O}_{X}
\end{array}\right)
$$

$$
\varphi((a, b),(s, \check{s}))=\left(\begin{array}{cc}
a & \mu s \\
\check{s} & b
\end{array}\right)
$$

for sections $a, b$ in $\mathscr{O}_{X}, s$ in $\mathscr{L}, \check{s}$ in $\check{\mathscr{L}}$, is easily seen to be an isomorphism of composition algebras. Q.E.D.

2.8 Remark. The invertible sheaf $\mathscr{L}$ in 2.7 is not uniquely determined by $\mathscr{C}$. In fact,

$$
\left(\begin{array}{cc}
\mathscr{O}_{X} & \mathscr{L} \\
\check{L}^{2} & \mathscr{O}_{X}
\end{array}\right) \stackrel{\rightarrow}{\rightarrow}\left(\begin{array}{cc}
\mathscr{O}_{X} & \check{\mathscr{L}} \\
\mathscr{L} & \mathscr{O}_{X}
\end{array}\right), \quad\left(\begin{array}{ll}
a & s \\
\check{s} & b
\end{array}\right) \mapsto^{t}\left(\begin{array}{ll}
a & s \\
\check{s} & b
\end{array}\right)^{*}=\left(\begin{array}{cc}
b & -\check{s} \\
-s & a
\end{array}\right)
$$

is an isomorphism.

2.9 Example. Let $\mathscr{D}$ be an associative composition algebra over the locally ringed space $X$. Then $\mathscr{D}_{\mathscr{D}}$, which is simply $\mathscr{D}$ viewed canonically as a right $\mathscr{D}$-module, is globally free of rank one and norm one. The norms on $\mathscr{D}_{\mathscr{D}}$ are exactly the quadratic forms $N=\mu N_{\mathscr{D}}, \mu \in \Gamma\left(X, \mathscr{O}_{X}^{\times}\right)$; the bilinear map 
$\mathscr{D}_{\mathscr{D}} \times \mathscr{D}_{\mathscr{D}} \rightarrow \mathscr{D}$ associated with such an $N$ is then given by $\left(w, w^{\prime}\right) \mapsto \mu w^{\prime *} w$, and the multiplication of $\operatorname{Cay}\left(\mathscr{D}, \mathscr{D}_{\mathscr{D}}, N\right)$ attains the form

$$
(u, w)\left(u^{\prime}, w^{\prime}\right)=\left(u u^{\prime}+\mu w^{\prime *} w, w^{\prime} u+w u^{\prime *}\right) .
$$

Hence we are back to the original Cayley-Dickson Doubling Process of 2.1.

2.10 Example. For $X, \mathscr{D}$ as in 2.9 , Pic $X$ acts on $\check{H}^{1}\left(X, \mathscr{D}^{\times}\right)$via $(\mathscr{L}, \mathscr{P})$ $\mapsto \mathscr{L} \otimes \mathscr{P}$, and we have $N_{\mathscr{D}}(\mathscr{L} \otimes \mathscr{P}) \cong \mathscr{L}^{2} \otimes N_{\mathscr{D}}(\mathscr{P})$. This allows us to construct examples of right $\mathscr{D}$-modules which are locally free of rank one and norm one without being globally free. For instance, if $\mathscr{L} \in \operatorname{Pic} X$ has order two, $\mathscr{L} \otimes \mathscr{D}_{\mathscr{D}}$ is such a module.

2.11 Example. Let $\mathscr{E}$ a locally free module of constant rank two over $X$. Then $\mathscr{D}=\mathscr{E}_{n} d_{X}(\mathscr{E})$, the $\mathscr{O}_{X}$-algebra of endomorphisms of $\mathscr{E}$, is a quaternion algebra over $X$ whose norm is the usual determinant (cf. EGA II 6.4.8). The canonical involution of $\mathscr{D}(1.6)$ may be described as follows: Recalling the natural isomorphism

$$
\Phi_{\mathscr{E}}:(\operatorname{det} \mathscr{E}) \otimes \check{\mathscr{E}} \stackrel{\sim}{\rightarrow} \mathscr{E} \quad\left(\operatorname{det} \mathscr{E}=\bigwedge^{2} \mathscr{E}\right)
$$

given by

$$
\boldsymbol{\Phi}_{\mathscr{E}}\left(\left(s_{1} \wedge s_{2}\right) \otimes \check{t}\right)=\left\langle s_{1}, \check{t}\right\rangle s_{2}-\left\langle s_{2}, \check{t}\right\rangle s_{1},
$$

we have

$$
f^{*}=\boldsymbol{\Phi}_{\mathscr{C}} \circ\left(\mathbf{1}_{\operatorname{det} \mathscr{E}} \otimes \check{f}\right) \circ\left(\boldsymbol{\Phi}_{\mathscr{C}}\right)^{-1}
$$

for $f$ in $\mathscr{D}$. Standard techniques in glueing local data on ringed spaces show that all locally free right $\mathscr{D}$-modules of rank one have the form

$$
\mathscr{P}=\mathscr{F} \otimes \check{\mathscr{E}}=\mathscr{H} m_{X}(\mathscr{E}, \mathscr{F}),
$$

$\mathscr{F}$ being a locally free $\mathscr{O}_{X}$-module of constant rank two; it follows $N_{\mathscr{D}}(\mathscr{P}) \cong$ $(\operatorname{det} \mathscr{F}) \otimes(\operatorname{det} \mathscr{E})^{\vee}$, so $\mathscr{P}$ has norm one if and only if there exists an isomorphism $\alpha: \operatorname{det} \mathscr{F} \stackrel{\sim}{\rightarrow} \operatorname{det} \mathscr{E}$. Fixing such an isomorphism, there exists a unique quadratic form $N: \mathscr{P} \rightarrow \mathscr{O}_{X}$ satisfying

$$
N(g)\left(s_{1} \wedge s_{2}\right)=\alpha\left(g\left(s_{1}\right) \wedge g\left(s_{2}\right)\right)
$$

for $g$ in $\mathscr{P}=\mathscr{H} a m(\mathscr{E}, \mathscr{F}), s_{1}, s_{2}$ in $\mathscr{E}$, and $N$ is a norm on $\mathscr{P}$ whose associated bilinear map $\mathscr{P} \times \mathscr{P} \rightarrow \mathscr{D}$ has the form

$$
\left(g_{1}, g_{2}\right) \mapsto g_{1}^{*} \circ g_{2},
$$

$g^{*}$, for $g$ in $\mathscr{P}$, being defined by

$$
g^{*}=\Phi_{\mathscr{C}} \circ(\alpha \otimes \check{g}) \circ\left(\Phi_{\mathscr{F}}\right)^{-1} .
$$

\section{ZORN ALGEBRAS}

3.1. Let $R$ be an arbitrary base ring. It is known since the work of Zorn [Zo] that, using the standard vector product $R^{3} \times R^{3} \rightarrow R^{3},(u, v) \mapsto u \times v$, on three-dimensional column space over $R$, the free $R$-module

$$
\operatorname{Zor}(R)=\left(\begin{array}{cc}
R & R^{3} \\
R^{3} & R
\end{array}\right)
$$


of rank 8 becomes an octonion algebra under the multiplication

$$
\left(\begin{array}{cc}
a & u \\
u^{\prime} & a^{\prime}
\end{array}\right)\left(\begin{array}{cc}
b & v \\
v^{\prime} & b^{\prime}
\end{array}\right)=\left(\begin{array}{cc}
a b+{ }^{t} u v^{\prime} & a v+b^{\prime} u-u^{\prime} \times v^{\prime} \\
b u^{\prime}+a^{\prime} v^{\prime}+u \times v & { }^{t} u^{\prime} v+a^{\prime} b^{\prime}
\end{array}\right)
$$

for $a, a^{\prime}, b, b^{\prime} \in R, u, u^{\prime}, v, v^{\prime} \in R^{3}$, called the Zorn algebra (of vector matrices) over $R$. As in the case of quaternions, the norm of $\operatorname{Zor}(R)$ is the determinant:

$$
\operatorname{det}\left(\begin{array}{cc}
a & u \\
u^{\prime} & a^{\prime}
\end{array}\right)=a a^{\prime}-{ }^{t} u u^{\prime} \text {. }
$$

If $R$ is a field, $\operatorname{Zor}(R)$ is the only split octonion algebra over $R$.

3.2. We wish to extend this construction from rings to locally ringed spaces. To this end, we consider a locally ringed space $X$ and a locally free $\mathscr{O}_{X}$-module $\mathscr{T}$ of constant rank 3 such that $\operatorname{det} \mathscr{T}=\bigwedge^{3} \mathscr{T} \cong \mathscr{O}_{X}$. Fixing an isomorphism $\alpha: \operatorname{det} \mathscr{T} \stackrel{\sim}{\rightarrow} \mathscr{O}_{X}$, unique up to an invertible factor in $\Gamma\left(X, \mathscr{O}_{X}\right)$, we obtain an induced bilinear map $\mathscr{T} \times \mathscr{T} \rightarrow \mathscr{T}$ according to the rule

$$
(u, v) \mapsto u \times v=\alpha(u \wedge v \wedge-) \text {. }
$$

This bilinear map is called the vector product on $\mathscr{T}$ since, locally, it just looks like the ordinary vector product. On the other hand, $\alpha$ uniquely determines an isomorphism $\beta: \operatorname{det} \check{\mathscr{T}} \stackrel{\sim}{\rightarrow} \mathscr{O}_{X}$, characterized by the condition

$$
\alpha\left(u_{1} \wedge u_{2} \wedge u_{3}\right) \beta\left(\check{u}_{1} \wedge \check{u}_{2} \wedge \check{u}_{3}\right)=\operatorname{det}\left(\left\langle u_{i}, \check{u}_{j}\right\rangle\right)
$$

for $u_{i}$ in $\mathscr{T}, \check{u}_{j}$ in $\check{\mathscr{T}}, 1 \leq i, j \leq 3$. Hence we also obtain a vector product $\check{\mathscr{T}} \times \check{\mathscr{T}} \rightarrow \mathscr{T}$, defined analogously to the one on $\mathscr{T}$, using $\beta$ rather than $\alpha$.

3.3. Keeping the notations of 3.2 , we contend that

$$
\operatorname{Zor}(\mathscr{T}, \alpha)=\left(\begin{array}{cc}
\mathscr{O}_{X} & \mathscr{T} \\
\mathscr{T} & \mathscr{O}_{X}
\end{array}\right)
$$

becomes an octonion algebra over $X$ under the multiplication

$$
\left(\begin{array}{cc}
a & u \\
\check{u} & a^{\prime}
\end{array}\right)\left(\begin{array}{cc}
b & v \\
\check{v} & b^{\prime}
\end{array}\right)=\left(\begin{array}{cc}
a a^{\prime}+\langle u, \check{v}\rangle & a v+b^{\prime} u-\check{u} \times \check{v} \\
b \check{u}+a^{\prime} \check{v}+u \times v & \langle v, \breve{u}\rangle=a^{\prime} b^{\prime}
\end{array}\right)
$$

whose norm is given by the determinant:

$$
\operatorname{det}\left(\begin{array}{cc}
a & u \\
\check{u} & a^{\prime}
\end{array}\right)=a a^{\prime}-\langle u, \check{u}\rangle .
$$

Indeed, one simply notes that, locally, $\operatorname{Zor}(\mathscr{T}, \alpha)$ looks like the Zorn algebra of 3.1 , and then invokes $1.7 .^{2}$

3.4. The octonion algebras obtained in 3.3 are called Zorn algebras (of vector matrices) over $X$. Their construction is functorial in the parameters involved: Suppose $\mathscr{T}, \mathscr{T}^{\prime}$ are locally free $\mathscr{O}_{X}$-modules of constant rank 3 , and $\alpha: \operatorname{det} \mathscr{T} \stackrel{\sim}{\rightarrow} \mathscr{O}_{X}, \alpha^{\prime}: \operatorname{det} \mathscr{T}^{\prime} \stackrel{\sim}{\rightarrow} \mathscr{O}_{X}$ are isomorphisms. Suppose further that $\varphi:(\mathscr{T}, \alpha) \rightarrow\left(\mathscr{T}^{\prime}, \alpha^{\prime}\right)$ is a morphism, i.e., an $\mathscr{O}_{X}$-linear map $\mathscr{T} \rightarrow \mathscr{T}^{\prime}$ having $\alpha^{\prime} \circ(\operatorname{det} \varphi)=\alpha$. Then $\varphi$ is bijective, and

$$
\left(\begin{array}{cc}
a & u \\
\check{u} & a^{\prime}
\end{array}\right) \mapsto\left(\begin{array}{cc}
a & \varphi(u) \\
\check{\varphi}^{-1}(\check{u}) & a^{\prime}
\end{array}\right)
$$

\footnotetext{
${ }^{2}$ The author is indebted to $\mathrm{O}$. Loos, who greatly simplified the original construction of these algebras.
} 
determines an isomorphism $\operatorname{Zor}(\mathscr{T}, \alpha) \stackrel{\sim}{\rightarrow} \operatorname{Zor}\left(\mathscr{T}^{\prime}, \alpha^{\prime}\right)$. Along similar lines,

$$
\left(\begin{array}{cc}
a & u \\
\check{u} & a^{\prime}
\end{array}\right) \mapsto{ }^{t}\left(\begin{array}{cc}
a & u \\
\check{u} & a^{\prime}
\end{array}\right)^{*}=\left(\begin{array}{cc}
a^{\prime} & -\check{u} \\
-u & a
\end{array}\right)
$$

turns out to be an isomorphism $\operatorname{Zor}(\mathscr{T}, \alpha) \stackrel{\sim}{\rightarrow} \operatorname{Zor}(\check{T}, \beta), \beta$ being defined as in 3.2 .

Zorn algebras are obviously split. The converse is also true, as we shall now prove.

3.5 Theorem. Let $\mathscr{C}$ be a split octonion algebra over the locally ringed space $X$. Then $\mathscr{C}$ is a Zorn algebra. More precisely, given a split torus $\mathscr{D} \subset \mathscr{C}$, there exist a locally free $\mathscr{O}_{X}$-module $\mathscr{T}$ of constant rank 3 , an isomorphism $\alpha: \operatorname{det} \mathscr{T} \stackrel{\sim}{\rightarrow} \mathscr{O}_{X}$ and an isomorphism $\mathscr{C} \stackrel{\sim}{\rightarrow} \operatorname{Zor}(\mathscr{T}, \alpha)$ sending $\mathscr{D}$ to the diagonal of $\operatorname{Zor}(\mathscr{T}, \alpha)$.

Proof. We proceed in three steps.

(1) Identifying $\mathscr{D}=\mathscr{O}_{X} \oplus \mathscr{O}_{X}$, one obtains, by passing to global sections, a complete orthogonal system $\left(c_{1}, c_{2}\right)$ of primitive idempotents in $D=\Gamma(X, \mathscr{D})$ $\subset C=\Gamma(X, \mathscr{C})$, giving rise to a Peirce decomposition

$$
\mathscr{C}=\mathscr{C}_{11} \oplus \mathscr{C}_{12} \oplus \mathscr{C}_{21} \oplus \mathscr{C}_{22}, \quad \mathscr{C}_{i i}=\mathscr{O}_{X} c_{i} \quad(i=1,2)
$$

with the usual properties (cf. [S, 3.4]). The $\mathscr{O}_{X}$-modules $\mathscr{C}_{12}, \mathscr{C}_{21}$ are locally free and dual to each other under $T=T_{\mathscr{C}}$, so both have constant rank 3 . The expression $T\left(u_{1} u_{2} u_{3}\right)$ being alternating in $u_{1}, u_{2}, u_{3} \in \mathscr{T}=\mathscr{C}_{12}$, we obtain a unique $\mathscr{O}_{X}$-linear map $\alpha: \operatorname{det} \mathscr{T} \rightarrow \mathscr{O}_{X}$ satisfying $\alpha\left(u_{1} \wedge u_{2} \wedge u_{3}\right)=T\left(u_{1} u_{2} u_{3}\right)$.

(2) We now consider the special case $X=\operatorname{Spec} R$, where $R$ is a local ring, put $t=t_{C}$ and contend that there is a basis $\left(e_{1}, e_{2}, e_{3}\right)$ of $C_{12}=\Gamma\left(X, \mathscr{C}_{12}\right)$ over $R$ such that

$$
t\left(e_{1} e_{2} e_{3}\right)=1=-t\left(\check{e}_{1} \check{e}_{2} \check{e}_{3}\right),
$$

$\left(\check{e}_{i}\right)$ being the $t$-dual basis of $C_{21}=\Gamma\left(X, \mathscr{C}_{21}\right)$ relative to $\left(e_{i}\right)$. If $R$ is a field, the assumption $t\left(u_{1} u_{2} u_{3}\right)=0$ for all $u_{1}, u_{2}, u_{3} \in C_{12}$ forces $C_{12}^{2}=0$ by nondegeneracy of $t$, and from $(u v) \check{w}+(u \check{w}) v=u(v \check{w}+\check{w} v)$ for $u, v \in C_{12}$, $\check{w} \in C_{21}$, we conclude $t(u \check{w}) v=t(v \check{w}) u$, in contradiction to $C_{12}$ having dimension 3; hence $t\left(u_{1} u_{2} u_{3}\right) \neq 0$ for some $u_{1}, u_{2}, u_{3} \in C_{12}$. If $R$ is arbitrary, passing to its residue class field now yields a basis $\left(e_{i}\right)$ of $C_{12}$ satisfying $t\left(e_{1} e_{2} e_{3}\right)=1$. Since, in addition, $e_{1} e_{2} \in C_{21}$ is perpendicular to $e_{1}, e_{2}$, this implies $e_{1} e_{2}=\check{e}_{3}$. We also have $e_{i} \check{e}_{j}=\delta_{i j} c_{1}, \check{e}_{i} e_{j}=\delta_{i j} c_{2}$, which first yields

$$
\left(\check{e}_{1} \check{e}_{2}\right) e_{1}=\check{e}_{1}\left(\check{e}_{2} e_{1}\right)+\check{e}_{1}\left(e_{1} \check{e}_{2}\right)-\left(\check{e}_{1} e_{1}\right) \check{e}_{2}=-\check{e}_{2}
$$

and then

$$
t\left(\check{e}_{1} \check{e}_{2} \check{e}_{3}\right)=t\left(\check{e}_{1} \check{e}_{2}, e_{1} e_{2}\right)=t\left(\left(\check{e}_{1} \check{e}_{2}\right) e_{1}, e_{2}\right)=-t\left(\check{e}_{2} e_{2}\right)=-1,
$$

as claimed.

(3) Returning to the general situation, it follows from (2) that $\alpha$ as defined in (1) is an isomorphism and

$$
\operatorname{det}\left(T\left(u_{i}, \check{u}_{j}\right)\right)=-T\left(u_{1} u_{2} u_{3}\right) T\left(\check{u}_{1} \check{u}_{2} \check{u}_{3}\right)
$$

for $u_{1}, u_{2}, u_{3}$ in $\mathscr{T}, \check{u}_{1}, \check{u}_{2}, \check{u}_{3}$ in $\check{\mathscr{T}}=\mathscr{C}_{21}$. Hence the vector product $\mathscr{T} \times \mathscr{T} \rightarrow \mathscr{T}$ coming from $\alpha$ via 3.2 agrees with the original multiplication 
of $\mathscr{C}$ restricted to $\mathscr{C}_{12}$, and the vector product $\check{\mathscr{T}} \times \mathscr{T} \rightarrow \mathscr{T}$ belonging to the isomorphism $\beta: \operatorname{det} \mathscr{\mathscr { T }} \stackrel{\sim}{\rightarrow} \mathscr{O}_{X}$ is the negative of the original multiplication of $\mathscr{C}$ restricted to $\mathscr{C}_{21}$. It now follows that the map $\Phi: \mathscr{C} \rightarrow \operatorname{Zor}(\mathscr{T}, \alpha)$ given by

$$
\Phi\left(a c_{1}+u+\check{u}+a^{\prime} c_{2}\right)=\left(\begin{array}{cc}
a & u \\
\check{u} & a^{\prime}
\end{array}\right)
$$

for $a, a^{\prime}$ in $\mathscr{O}_{X}, u$ in $\mathscr{T}=\mathscr{C}_{12}, \check{u}$ in $\check{\mathscr{T}}=\mathscr{C}_{21}$ is an isomorphism of the desired kind. Q.E.D.

3.6 Corollary (cf. [BS, (3.4)]). Let $R$ be a principal ideal domain and $C$ a composition algebra with zero divisors over $R$. Then $C$ is isomorphic to $R \oplus R$, $\operatorname{Mat}_{2}(R)$ (the algebra of 2-by-2 matrices over $R$ ) or $\operatorname{Zor}(R)$.

Proof. Since finitely generated projective $R$-modules are free, and in view of $2.7,3.5$, it suffices to show that $C$ is split. So let $u \neq 0$ be a zero divisor in $C$. Then $n_{C}(u)=0$, and we may assume that $u$ is unimodular relative to some basis of $C$. By nondegeneracy, this yields an element $v \in C$ satisfying $t_{C}(u v)=1$, and $\left(u v, 1_{C}-u v\right)$ is a complete orthogonal system of primitive idempotents in $C$. Q.E.D.

3.7. Let $X=\mathrm{P}_{R}^{n}$ be projective $n$-space over a ring $R$, so $X=\operatorname{Proj} S$ where $S=R\left[x_{0}, \ldots, x_{n}\right]$ is the polynomial ring in $n+1$ variables over $R$, equipped with the natural grading $S=\bigoplus_{d \geq 0} S_{d}$. Note that $X$ is covered by the open affines $U_{i}=\operatorname{Spec} S_{x_{i}}$ 。 for $i=0, \ldots, n$, where

$$
S_{x_{i} \circ}=R\left[\frac{x_{j}}{x_{i}} ; 0 \leq j \leq n, j \neq i\right]
$$

stands for the elements of degree 0 in the Z-graded $k$-algebra $S_{x_{i}}$ obtained by localizing $S$ with respect to the multiplicative subset $\left\{1, x_{i}, x_{i}^{2}, \ldots\right\}$. For $d \in \mathbf{Z}$, we write as usual $\mathscr{O}_{X}(d)$ for the unique (invertible) $\mathscr{O}_{X}$-module whose sections over $U_{i} \quad(0 \leq i \leq n)$ are the elements of degree $d$ in $S_{x_{i}}$. Recall that $\Gamma\left(X, \mathscr{O}_{X}(d)\right)$ vanishes for $d<0$ and agrees with $S_{d}$ otherwise [Ha, II (5.13)].

3.8. We now make good on our promise to construct natural examples of quadratic alternative algebras with big radicals (1.8). Keeping the above notations and fixing positive integers $l, m$,

$$
\mathscr{T}=\mathscr{O}_{X}(l) \oplus \mathscr{O}_{X}(m) \oplus \mathscr{O}_{X}(-l-m)
$$

is a locally free $\mathscr{O}_{X}$-module of constant rank 3 , and the multiplication of $S$ induces a canonical isomorphism $\alpha$ : $\operatorname{det} \mathscr{T} \stackrel{\sim}{\rightarrow} \mathscr{O}_{X}$. Hence we may form the octonion algebra $\mathscr{C}=\operatorname{Zor}(\mathscr{T}, \alpha)$ over $X$. From 3.7, we conclude

$$
C=\Gamma(X, \mathscr{C})=\left(\begin{array}{cc}
R & S_{l} \oplus S_{m} \\
S_{l+m} & R
\end{array}\right) .
$$

Multiplication in this $R$-algebra is carried out according to the rule

$$
\begin{aligned}
& \left(\begin{array}{cc}
a & f_{l} \oplus f_{m} \\
f_{l+m} & a^{\prime}
\end{array}\right)\left(\begin{array}{cc}
b & g_{l} \oplus g_{m} \\
g_{l+m} & b^{\prime}
\end{array}\right) \\
& \quad=\left(\begin{array}{cc}
a b & \left(a g_{l}+b^{\prime} f_{l}\right) \oplus\left(a g_{m}+b^{\prime} f_{m}\right) \\
b f_{l+m}+a^{\prime} g_{l+m}+f_{l} g_{m}-f_{m} g_{l} & a^{\prime} b^{\prime}
\end{array}\right),
\end{aligned}
$$


where $a, a^{\prime}, b, b^{\prime} \in R$ and the $f^{\prime} s$ and $g^{\prime} s$ are homogeneous polynomials in $S$, with subscripts indicating their respective degrees. $C$ is quadratic alternative (1.7) and free of rank

$$
\left(\begin{array}{c}
l+n \\
n
\end{array}\right)+\left(\begin{array}{c}
m+n \\
n
\end{array}\right)+\left(\begin{array}{c}
l+m+n \\
n
\end{array}\right)+2 .
$$

Note that $C$ is an $R$-subalgebra of $\operatorname{Zor}(S)$. If $R$ is a field, the radical of $C$ has the form

$$
\operatorname{rad} C=\left(\begin{array}{cc}
0 & S_{l} \oplus S_{m} \\
S_{l+m} & 0
\end{array}\right)
$$

and satisfies

$$
(\operatorname{rad} C)^{2}=\left(\begin{array}{cc}
0 & 0 \\
S_{l+m} & 0
\end{array}\right), \quad(\operatorname{rad} C)^{3}=0
$$

the last equation being part of a general phenomenon, observed independently by Zagler [Z, 6.4] and Kunze-Scheinberg [KS, Theorem 1.5], see also [M, 5.6].

\section{Curves of genus zero and the Classification Theorem}

4.1. Throughout this section, we fix an arbitrary base field $k$. By a curve we mean a geometrically integral, complete, smooth scheme of dimension one over $k$. Given a divisor $D$ on a curve $X$, we write $\operatorname{deg} D$ for its degree and $\mathscr{L}(D)$ for the corresponding invertible sheaf, so $D \mapsto \mathscr{L}(D)$ determines the canonical identification of the class group of $X$ with Pic $X$. The term "point" without further specification always refers to closed points. On the other hand, the generic point of $X$ is usually denoted by $\xi$. We write $\kappa(X)=\mathscr{O}_{\xi, X}$ for the function field of $X$.

4.2. Let $X$ be a curve of genus zero, i.e., a smooth quadric in the plane. The following statements are standard consequences of the Riemann-Roch Theorem (cf. [T]). $X$ always contains points of degree at most two and is rational (i.e., isomorphic to $\mathbf{P}_{k}^{1}$ ) if and only if it contains rational points. In particular, $X$ is a form of the projective line, so becomes isomorphic to it after passing to the algebraic closure or, in fact, to an appropriate quadratic extension. If $P_{0} \in X$ is a point of minimal degree $(\leq 2)$, the assignment $m \mapsto \mathscr{L}\left(m P_{0}\right)$ gives an isomorphism $\mathrm{Z} \stackrel{\sim}{\rightarrow} \mathrm{Pic} X$. We also have

$$
\operatorname{dim}_{k} \Gamma(X, \mathscr{L}(D))=\operatorname{deg} D+1
$$

for every divisor $D$ of $X$ having degree $\geq 0$, whereas $\Gamma(X, \mathscr{L}(D))=0$ otherwise.

4.3. Following Witt [W], there is a one-to-one correspondence between (nonrational) curves of genus zero and quaternion (division) algebras over $k$. In order to describe this correspondence, we adopt the approach of Tillmann [T, 5.4]. Accordingly, $X$ being a nonrational curve of genus zero, there exists an indecomposable vector bundle $\mathscr{E}$ of rank two over $X$, unique up to multiplication with a unique invertible sheaf, and $D=\operatorname{End}_{X}(\mathscr{E})$ is the quaternion division algebra we are looking for. A standard model $\mathscr{E}_{0}$ of such a bundle may be described as follows: Let $P_{0} \in X$ be a point of degree two, $t$ a local parameter of $X$ at $P_{0}$ and $f \in \mathscr{O}_{P_{0}, X}^{\times}$have the property that $f\left(P_{0}\right)$ generates $\kappa\left(P_{0}\right) / k$. Then $\mathscr{E}_{0}$ is the subsheaf of the constant sheaf $\boldsymbol{\kappa}(X)^{2}$ (column vectors) which 
agrees with $\mathscr{O}_{X}^{2}$ outside $P_{0}$ and whose stalk at $P_{0}$ is the (free) $\mathscr{O}_{P_{0}}$-module generated by $\left(\begin{array}{l}1 \\ 0\end{array}\right),\left(\begin{array}{c}t^{-1} f \\ t^{-1}\end{array}\right)$. This description obviously implies $\operatorname{det} \mathscr{E}_{0}=\mathscr{L}\left(P_{0}\right)$. We also recall from $[\mathrm{T}, 5.5]$ that all locally free $\mathscr{O}_{X}$-modules of rank at least 3 are decomposable.

Now we are in a position to state the main results of the paper.

4.4 Classification Theorem. Let $X$ be a curve of genus zero and $\mathscr{C}$ a composition algebra over $X$. Then one of the following holds.

(i) $\mathscr{C}$ is defined over $k$ (see also 4.6).

(ii) $\mathscr{C}$ is a split quaternion algebra (see also 4.7).

(iii) $\mathscr{C}$ is a split octonion algebra (see also 4.8 ).

(iv) $X$ is not rational and $\mathscr{C} \cong \operatorname{Cay}(\mathscr{D}, \mathscr{P}, N)$, where $\mathscr{D}$ is the base change from $k$ to $X$ of the quaternion division algebra over $k$ associated with $X, \mathscr{P}$ is a locally free right $\mathscr{D}$-module of rank one and norm one, and $N$ is a norm on $\mathscr{P}$ (see also 4.9).

4.5 Theorem. Let $X$ be a curve of genus zero and $\mathscr{C}$ a composition algebra of rank $r$ over $X$. Suppose $\mathscr{C}$ contains a nonsplit composition subalgebra of rank $\frac{r}{2}$ which is defined over $k$ but not isomorphic to the base change from $k$ to $X$ of the quaternion algebra over $k$ associated with $X$. Then $\mathscr{C}$ is defined over $k$.

4.6. A few comments on the Classification Theorem, whose proof, along with the one of 4.5 , will be postponed to the next section, are in order. Let $Y$ be any $k$-scheme having $\Gamma\left(Y, \mathscr{O}_{Y}\right)=k$ and $C$ a composition algebra over $k$. Writing $\mathscr{C}$ for the base change of $C$ from $k$ to $Y$, we conclude that the natural map $\rho: C \rightarrow \Gamma(Y, \mathscr{C})$ is an isomorphism since both algebras have the same dimension, $C$ is simple as an algebra with involution and $\rho$ preserves involutions. In particular, we recover $C$ from $\mathscr{C}$ by passing to global sections, which, in the situation of 4.4 , implies that composition algebras over $X$ defined over $k$ are basically the same as composition algebras over $k$.

4.7. Let $\mathscr{C}$ be a split quaternion algebra over $X$ and suppose $\mathscr{C}$ is not defined over $k$. Then, by 2.7 and 4.2 ,

$$
\mathscr{C} \cong\left(\begin{array}{cc}
\mathscr{O}_{X} & \mathscr{L}\left(m P_{0}\right) \\
\mathscr{L}\left(-m P_{0}\right) & \mathscr{O}_{X}
\end{array}\right),
$$

where $P_{0} \in X$ is a point of minimal degree $(\leq 2)$ and $m \in \mathrm{Z}$ is nonzero; in fact, we may even assume $m>0$ (2.8). Then the Krull-Schmidt Theorem [T, Satz 2.7], according to which any locally free $\mathscr{O}_{X}$-module splits into the direct sum of indecomposables unique up to order and isomorphism, shows that $m$ is uniquely determined by $\mathscr{C}$.

4.8. Now let $\mathscr{C}$ be a split octonion algebra over $X$ and again suppose $\mathscr{C}$ is not defined over $k$. Then, by $3.5, \mathscr{C}$ is a Zorn algebra over $X$, so $\mathscr{C} \cong$ $\operatorname{Zor}(\mathscr{T}, \alpha)$, for some locally free $\mathscr{O}_{X}$-module $\mathscr{T}$ of rank 3 and some isomorphism $\alpha: \operatorname{det} \mathscr{T} \stackrel{\sim}{\rightarrow} \mathscr{O}_{X}$. With $P_{0}$ as in 4.7 , we then have the following possibilities for $\mathscr{T}$.

Case 1. $\mathscr{T}$ splits into the direct sum of line bundles. Then, since $\operatorname{det} \mathscr{T} \cong \mathscr{O}_{X}$,

$$
\mathscr{T}=\mathscr{L}\left(m_{1} P_{0}\right) \oplus \mathscr{L}\left(m_{2} P_{0}\right) \oplus \mathscr{L}\left(-\left(m_{1}+m_{2}\right) P_{0}\right)
$$


for integers $m_{1}, m_{2}$ not both zero; in fact, using functoriality (3.4), we may assume $m_{1} \geq m_{2} \geq 0, m_{1}>0$ and then conclude from the Krull-Schmidt Theorem that $\mathscr{C}$ determines $m_{1}, m_{2}$ uniquely. Analogously, the isomorphism class of $\mathscr{C}$ is easily seen to be independent of the choice of $\alpha$.

Case 2. $\mathscr{T}$ does not split into the direct sum of line bundles. Then $X$ is not rational and, arguing as in Case 1, we conclude

$$
\mathscr{T}=\mathscr{L}\left(-(2 m+1) P_{0}\right) \oplus\left[\mathscr{L}\left(m P_{0}\right) \otimes \mathscr{E}_{0}\right],
$$

where $\mathscr{E}_{0}$ has the meaning explained in 4.3 and $m \geq 0$ is an integer uniquely determined by $\mathscr{C}$. Again $\mathscr{C}$ is independent of the choice of $\alpha$.

4.9. Finally, suppose $X$ is not rational and write $D$ for the quaternion division algebra over $k$ associated with $X$. For $\mathscr{D}, \mathscr{P}, N$ as in 4.4(iv), we assume that $\mathscr{C}=\operatorname{Cay}(\mathscr{D}, \mathscr{P}, N)$ is not defined over $k$. Observing $D=\operatorname{End}_{X}\left(\mathscr{E}_{0}\right)$ (4.3), 5.2 below shows $\mathscr{D}=\mathscr{C}_{n} d_{X}\left(\mathscr{E}_{0}\right)$. As in 2.11 , this gives $\mathscr{P}=\mathscr{F} \otimes \check{\mathscr{C}}_{0}=$ $\mathscr{H} m_{X}\left(\mathscr{E}_{0}, \mathscr{F}\right)$, where $\mathscr{F}$ is a locally free $\mathscr{O}_{X}$-module of rank two satisfying $\operatorname{det} \mathscr{F} \cong \operatorname{det} \mathscr{E}_{0}=\mathscr{L}\left(P_{0}\right)$. If $\mathscr{F}$ were indecomposable, this would imply $\mathscr{F}=$ $\mathscr{E}_{0}$, hence $\mathscr{P}=\mathscr{D}_{\mathscr{D}}$, forcing $\mathscr{C}$ to be defined over $k$ (1.10), a contradiction. Hence $\mathscr{F}$ splits and so, without loss, $\mathscr{P}$ attains its final form

$$
\mathscr{P}=\mathscr{P}_{1} \oplus \mathscr{P}_{2}, \quad \mathscr{P}_{1}=\mathscr{L}\left(m P_{0}\right) \otimes \check{\mathscr{E}}_{0}, \quad \mathscr{P}_{2}=\mathscr{L}\left((-m+1) P_{0}\right) \otimes \check{\mathscr{E}}_{0}
$$

for some integer $m \geq 0$ uniquely determined by $\mathscr{C}$. Viewing $\check{\mathscr{E}}_{0}$ as a sheaf of row vectors, the norm $N_{0}$ on $\mathscr{P}$ determined by the natural isomorphism $\alpha: \operatorname{det} \mathscr{F} \stackrel{\sim}{\rightarrow} \operatorname{det} \mathscr{E}_{0}$ via 2.11 has $\mathscr{P}_{i}(i=1,2)$ as totally isotropic submodules and induces the canonical pairing

$$
\left(s_{1} \otimes \check{t}_{1}, s_{2} \otimes \check{t}_{2}\right) \mapsto-s_{1} s_{2} \operatorname{det}\left(\begin{array}{c}
\check{t}_{1} \\
\check{t}_{2}
\end{array}\right)
$$

on $\mathscr{P}_{1} \times \mathscr{P}_{2}$. We now conclude $N=\mu N_{0}$ for some $\mu \in k^{\times}$(2.4). Also, the map $(\mathscr{P}, N) \rightarrow\left(\mathscr{P}, N_{0}\right)$ defined to be multiplication by $\mu$ on the first summand and the identity on the second turns out to be a morphism in the sense of 2.6 and hence shows $\mathscr{C}=\operatorname{Cay}(\mathscr{D}, \mathscr{P}, N) \cong \operatorname{Cay}\left(\mathscr{D}, \mathscr{P}, N_{0}\right)$.

Using the Krull-Schmidt Theorem, the octonion algebras constructed in 4.8, Cases 1,2 , and 4.9 are easily seen to be mutually nonisomorphic. We have thus obtained a complete classification, without repetitions, of composition algebras over $X$.

\section{Proofs}

5.1. The proofs of $4.4,4.5$, which are our main concern in this section, will be carried out simultaneously. We continue to work over an arbitrary base field $k$ and consider a fixed curve $X$ of genus zero. Our first objective is to show that every composition algebra over $X$, other than $\mathscr{O}_{X}$, contains a torus defined over $k$. To this end, we first require the following elementary but crucial observation, which, incidentally, generalizes 1.10 .

5.2 Proposition. Let $R$ be a ring, $Y$ an $R$-scheme, $\mathscr{C}$ a quadratic algebra over $Y$ and $D \subset \Gamma(Y, \mathscr{C})$ a composition subalgebra over $R$ having $\Gamma\left(Y, \mathscr{O}_{Y}\right)=R$.

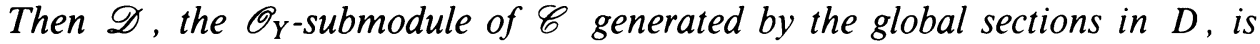


a composition subalgebra defined over $R$. More precisely, there is a natural isomorphism from $\mathscr{D}$ to the base change of $D$ from $R$ to $Y$.

Proof. We write $\sigma: Y \rightarrow Z=\operatorname{Spec} R$ for the structure morphism of $Y$ and denote by $M \mapsto \widetilde{M}$ the natural equivalence between the category of $R$-modules and the category of quasicoherent $\mathscr{O}_{Z}$-modules. Recall that, more generally, the functor $\sim$ is left adjoint to the global section function $\Gamma(Z, \cdot)$ from $\mathscr{O}_{Z^{-}}$ modules to $R$-modules and, also, that $\sigma^{*}$ is left adjoint to the direct image functor $\sigma_{*}$ from $\mathscr{O}_{Y}$-modules to $\mathscr{O}_{Z}$-modules. This being so, the inclusion $D \hookrightarrow$ $\Gamma(Y, \mathscr{C})=\Gamma\left(Z, \sigma_{*} \mathscr{C}\right)$ first induces a homomorphism $\widetilde{D} \rightarrow \sigma_{*} \mathscr{C}$ and then a homomorphism $\alpha: \sigma^{*} \widetilde{D} \rightarrow \mathscr{C}$ of quadratic algebras whose image is $\mathscr{D}$. For each $P \in Y$ the map $\left(\sigma^{*} \widetilde{D}\right) \otimes \boldsymbol{\kappa}(P) \rightarrow \mathscr{C} \otimes \boldsymbol{\kappa}(P)$ determined by $\alpha$ is injective since composition algebras over fields are simple as algebras with involution. Hence $\alpha$ is injective and so yields an isomorphism $\sigma^{*} \widetilde{D} \stackrel{\sim}{\rightarrow} \mathscr{D}$. Q.E.D.

5.3 Corollary. Let $Y$ be a projective $k$-scheme having $\Gamma\left(Y, \mathscr{O}_{Y}\right)=k, \mathscr{C}$ a composition algebra over $Y$ and $k^{\prime} / k$ an arbitrary field extension. Write $\mathscr{C}^{\prime}$ for the base change of $\mathscr{C}$ from $Y$ to $Y^{\prime}=Y \times_{k} k^{\prime}$. If $\mathscr{C}^{\prime}$ admits a composition subalgebra of rank $r$ defined over $k^{\prime}$, then $\mathscr{C}$ admits a composition subalgebra of rank $r$ defined over $k$.

Proof. $C=\Gamma(Y, \mathscr{C}), C^{\prime}=\Gamma\left(Y^{\prime}, \mathscr{C}^{\prime}\right)$ are finite-dimensional quadratic alternative algebras over $k, k^{\prime}$, respectively (1.7), and we have $C^{\prime}=C \otimes_{k} k^{\prime}$. By hypothesis, $C^{\prime}$ contains a composition subalgebra of dimension $r$ over $k^{\prime}$. Since the assertion is trivial for $r=1$, we may assume $r>1$. Then $C$ becomes a composition algebra modulo its radical and so contains a Wedderburn factor [S, Theorem 3.18], which is a composition algebra of rank at least $r$, hence admits a composition subalgebra of rank exactly $r$. Now 5.2 applies. Q.E.D.

5.4. At this stage we allow ourselves a digression. Letting $Y=\mathbf{F}_{k}^{11}$ be the projective line over $k$, we wish to classify up to isometry quadratic spaces over $Y$, i.e., pairs $(\mathscr{E}, Q)$ where $\mathscr{E}$ is a locally free $\mathscr{O}_{Y}$-module of finite rank and $Q: \mathscr{E} \rightarrow \mathscr{O}_{Y}$ is a quadratic form whose induced symmetric bilinear form is nondegenerate. For bilinear spaces, hence for quadratic ones as well if the characteristic is not two, this classification has been carried out by Knebusch [K, Theorem 13.2.2]. With very minor adjustments, however, the same argument works for quadratic spaces over an arbitrary base field, leading to a result which may be described as follows. For a positive integer $m$, the hyperbolic quadratic form $(a, b) \mapsto a b$ gives the $\mathscr{O}_{X}$-module $\mathscr{O}_{X}(m) \oplus \mathscr{O}_{X}(-m)$ the structure of a quadratic space over $Y$, which we denote by $\mathbf{h}(m)$; quadratic spaces of this kind are called hyperbolic planes. Knebusch's Theorem now says that a quadratic space over $Y$ decomposes into the orthogonal sum of finitely many hyperbolic planes and a quadratic space defined over $k$.

Returning to our original curve $X$ of genus zero, we are now in a position to establish an auxiliary result which is implicit in the Classification Theorem 4.4 and turns out to be of critical importance for the completion of the proof.

5.5 Lemma (cf. [H, Satz 3.1]). Let $\mathscr{C}$ be a composition algebra over $X$ having rank $2^{n}>1$. Then $\mathscr{C}$ contains a torus defined over $k$.

Proof. By 5.3 we may assume that $X=\mathbf{P}_{k}^{1}$ is the projective line over $k$. Writing $N=N_{\mathscr{C}}$ for the norm of $\mathscr{C}$, Knebusch's Theorem (5.4) allows us to 
decompose the quadratic space $(\mathscr{C}, N)$ as

$$
(\mathscr{C}, N)=\mathbf{h}\left(m_{1}\right) \perp \cdots \perp \mathbf{h}\left(m_{r}\right) \perp \sigma^{*}(V, q),
$$

where $0 \leq r \leq 2^{n-1}, m_{1} \geq \cdots \geq m_{r}>0$ are integers, $\sigma: X \rightarrow$ Spec $k$ denotes the structure morphism, and $(V, q)$ is a quadratic space over $k$. Passing to global sections and observing 3.7, we conclude

$$
C=\Gamma(X, \mathscr{C})=\Gamma\left(X, \mathscr{O}_{X}\left(m_{1}\right)\right) \perp \cdots \perp \Gamma\left(X, \mathscr{O}_{X}\left(m_{r}\right)\right) \perp V
$$

and

$$
1_{C}=\sum_{j=1}^{r} g_{j}+e
$$

for some $g_{j} \in S$ homogeneous of degree $m_{j}$ and $e \in V$. Hence $q(e)=$ $N\left(1_{C}\right)=1$, so $r<2^{n-1}$, and $V$ has dimension at least two. It is now easy to find a vector $u \in V, u \notin k e$, such that the subspace of $V$ spanned by $e, u$ is nondegenerate relative to the symmetric bilinear form induced by $q$. Thus $k 1_{C}+k u=k[u] \subset C$ is a composition subalgebra, giving rise to a subtorus of $\mathscr{C}$ defined over $k$ (5.2). Q.E.D.

5.6 Remark. 5.5 says in particular that every torus over $X$ is defined over $k$. For $k$ algebraically closed of characteristic not two, this result may be put into a more general context as follows. Let $Y$ be any curve over $k$. Then a torus over $Y$ has the form $\operatorname{Cay}\left(\mathscr{O}_{Y}, \mathscr{L}, N\right)$ where $\mathscr{L} \in \operatorname{Pic} Y$ has order two and $N$ is a norm on $\mathscr{L}(2.5)$. Hence any torus over $Y$ determines an element of order two in Pic $Y$; equivalently, it determines an étale cover of $Y$ having degree two [Ha, IV Ex 2.7]. Since Pic $X \cong Z$ is torsionfree, and $X$ is simply connected, both of these facts explain why any torus over $X$ is defined over $k$.

5.7. Before proceeding with the proof, we introduce some notation. Let $k^{\prime} / k$ be a separable quadratic field extension. If $\mathscr{C}$ is a composition algebra over $X$, we write $\mathscr{C}^{\prime}$ for its base change from $X$ to $X^{\prime}=X \times_{k} k^{\prime}$; similar conventions apply to other algebraic objects over $X$. The nontrivial Galois automorphism of $k^{\prime} / k$ will be denoted by $\tau$. We let it act on $X^{\prime}$ through the second factor. Observe that, given any $\mathscr{O}_{X}$-module $\mathscr{F}, \tau$ acts on $\mathscr{F}^{\prime}$; more precisely, we obtain a natural isomorphism $\tau^{*} \mathscr{F}^{\prime} \stackrel{\sim}{\rightarrow} \mathscr{F}^{\prime}$. Also, the automorphism of Pic $X^{\prime}$ induced by $\tau$ is the identity.

5.8. Now suppose $\mathscr{C}$ is a quaternion algebra over $X$. Then $\mathscr{C}$ contains a torus $\mathscr{D}$ defined over $k$ (5.5), and, in order to prove $4.4,4.5$ for $\mathscr{C}$, we may assume that $\mathscr{D}$ is not split, so it comes from a separable quadratic field extension $k^{\prime} / k$ by changing scalars from $k$ to $X$. We have to show that $\mathscr{C}$ is defined over $k$. Adopting the terminology of 5.7, it suffices to show that $\mathscr{C}^{\prime}$ is defined over $k^{\prime}$ (5.3). But, $\mathscr{D}^{\prime}$ being a split torus in $\mathscr{C}^{\prime}, 2.7$ yields an identification

$$
\mathscr{C}^{\prime}=\left(\begin{array}{ll}
\mathscr{O}_{X^{\prime}} & \mathscr{L}^{\prime} \\
\check{\mathscr{L}}^{\prime} & \mathscr{O}_{X^{\prime}}
\end{array}\right)
$$

matching $\mathscr{D}^{\prime}$ with the diagonal, where $\mathscr{L}^{\prime}$ is an invertible sheaf on $X^{\prime}$. Therefore $\tau$, being the exchange involution on $\mathscr{D}^{\prime}$, hence switching the diagonal idempotents in the matrix representation of $\mathscr{C}^{\prime}$ above, induces an isomorphism $\mathscr{L}^{\prime} \stackrel{\sim}{\rightarrow} \check{\mathscr{L}}^{\prime}$. Since $\mathrm{Pic} X^{\prime} \cong \mathrm{Z}$ is torsionfree, this forces $\mathscr{L}^{\prime} \cong \mathscr{O}_{X^{\prime}}$, making $\mathscr{C}^{\prime}$ globally free as an $\mathscr{O}_{X^{\prime}}$-module, hence defined over $k^{\prime}$ (1.10). Q.E.D. 
5.9. It remains to establish $4.4,4.5$ for an octonion algebra $\mathscr{C}$ over $X$. Assuming $\mathscr{C}$ to be nonsplit, our first goal is to show that $\mathscr{C}$ contains a quaternion subalgebra defined over $k$. To this end, we again start with a torus $\mathscr{D} \subset \mathscr{C}$ defined over $k$ which agrees with the base change from $k$ to $X$ of a separable quadratic field extension $k^{\prime} / k$. Again adopting the terminology of 5.7, it suffices to show that $\mathscr{C}^{\prime}$ contains a quaternion subalgebra defined over $k^{\prime}$. But $\mathscr{C}^{\prime}$, containing $\mathscr{D}^{\prime}$ as a split torus, is itself split and so is a Zorn algebra over $X^{\prime}$ as in 3.3: $\mathscr{C}^{\prime}=\operatorname{Zor}\left(\mathscr{T}^{\prime}, \alpha^{\prime}\right)$ where $\mathscr{T}^{\prime}$ is a locally free $\mathscr{O}_{X^{\prime}}$-module of rank 3 and $\alpha^{\prime}: \operatorname{det} \mathscr{T}^{\prime} \stackrel{\sim}{\rightarrow} \mathscr{O}_{X^{\prime}}$ is an isomorphism. Again $\tau$ interchanges the diagonal and so yields an isomorphism $\tau^{*} \mathscr{T}^{\prime} \stackrel{\sim}{\rightarrow} \mathscr{T}^{\prime}$. Now write $\mathscr{T}^{\prime}=\mathscr{L}^{\prime} \oplus \mathscr{T}_{1}^{\prime}$, where $\mathscr{L}^{\prime}, \mathscr{T}_{1}^{\prime}$ are locally free of rank 1,2 , respectively. If $\mathscr{T}_{1}^{\prime}$ were indecomposable, the Krull-Schmidt Theorem would imply $\mathscr{L}^{\prime} \cong \mathscr{O}_{X^{\prime}}$, hence $\operatorname{det} \mathscr{T}_{1}^{\prime} \cong \operatorname{det} \mathscr{T}^{\prime} \cong \mathscr{O}_{X^{\prime}}$, a contradiction. Therefore $\mathscr{T}^{\prime}$ is a self-dual direct sum of line bundles and so has the form $\mathscr{T}^{\prime}=\mathscr{L}^{\prime} \oplus \mathscr{O}_{X^{\prime}} \oplus \check{\mathscr{L}}^{\prime}$, for some invertible sheaf $\mathscr{L}^{\prime}$ on $X^{\prime}$. This gives $\mathscr{T}^{\prime}=\check{\mathscr{L}}^{\prime} \oplus \mathscr{O}_{X^{\prime}} \oplus \mathscr{L}^{\prime}$, and the two middle summands of $\mathscr{T}^{\prime}, \mathscr{T}^{\prime}$, together with the diagonal $\mathscr{D}^{\prime} \subset \mathscr{C}^{\prime}$, produce a quaternion subalgebra of $\mathscr{C}^{\prime}$ defined over $k^{\prime}$, as claimed.

5.10. Continuing with the proof of $4.4,4.5$ for our nonsplit octonion algebra $\mathscr{C}$ over $X$, we change notation and now let $\mathscr{D} \subset \mathscr{C}$ be a quaternion subalgebra defined over $k(5.9)$, so $\mathscr{D}=\sigma^{*} D$ for some quaternion algebra $D$ over $k$, $\sigma: X \rightarrow$ Spec $k$ being the structure morphism. We may assume that $D$ is not split and not the quaternion algebra associated with $X$ and have to show that $\mathscr{C}$ is defined over $k$. Since $\boldsymbol{\kappa}(X)$, the function field of $X$, splits $X$ without splitting $\mathscr{D}[W]$, we may assume $X=\mathbf{P}_{k}^{1}$. Now write $\mathscr{C}=\operatorname{Cay}(\mathscr{D}, \mathscr{P}, N)$ as in 2.5 and consider a separable quadratic field extension $k^{\prime} / k$ that splits $D$. Adopting the terminology of 5.7, we obtain $\mathscr{C}^{\prime}=\operatorname{Cay}\left(\mathscr{D}^{\prime}, \mathscr{P}^{\prime}, N^{\prime}\right)$ and $\mathscr{D}^{\prime}=\mathscr{E}_{n} d_{X^{\prime}}\left(\mathscr{O}_{X^{\prime}} \oplus \mathscr{O}_{X^{\prime}}\right)$, so $\mathscr{P}^{\prime}=\mathscr{O}_{X^{\prime}}(m) \oplus \mathscr{O}_{X^{\prime}}(m) \oplus \mathscr{O}_{X^{\prime}}(-m) \oplus \mathscr{O}_{X^{\prime}}(-m)$ for some integer $m \geq 0$ as an $\mathscr{O}_{X^{\prime}}$-module $(2.11)$, forcing $\mathscr{P}=\mathscr{O}_{X}(m) \oplus \mathscr{O}_{X}(m) \oplus$ $\mathscr{O}_{X}(-m) \oplus \mathscr{O}_{X}(-m)$ as an $\mathscr{O}_{X}$-module. By 1.10 it suffices to show $m=0$. Arguing indirectly, we assume $m>0$. The right $\mathscr{D}$-module structure of $\mathscr{P}$ amounts to a homomorphism $\mathscr{D}$ op $\rightarrow \mathscr{E}_{n d} d_{X}(\mathscr{P})$ of $\mathscr{O}_{X}$-algebras. By passing to global sections and observing 3.7 in conjunction with the natural isomorphisms

$$
\mathscr{H} a m\left(\mathscr{O}_{X}(p), \mathscr{O}_{X}(q)\right) \cong \mathscr{O}_{X}(p)^{\vee} \otimes \mathscr{O}_{X}(q) \cong \mathscr{O}_{X}(q-p)
$$

for arbitrary integers $p, q$, this gives rise to a homomorphism

$$
\varphi: D^{\text {op }} \rightarrow\left(\begin{array}{cc}
\operatorname{Mat}_{2}(k) & \operatorname{Mat}_{2}\left(S_{2 m}\right) \\
0 & \operatorname{Mat}_{2}(k)
\end{array}\right)
$$

of $k$-algebras, where $\mathrm{Mat}_{2}$ refers to $2 \times 2$-matrices and $S=\bigoplus_{d \geq 0} S_{d}$ is the polynomial ring in two variables over $k$ with its natural grading. Following $\varphi$ with the projection to the upper left-hand corner of the block matrices on the right now yields a homomorphism $D^{\text {op }} \rightarrow \operatorname{Mat}_{2}(k)$. Since $D$ is a division algebra, this cannot be. Q.E.D.

\section{Applications}

6.1. We now apply our previous results to one-dimensional function fields of genus zero. In doing so, we will always tacitly assume that the nonsingular model 
associated with such a field is a curve in the sense of 4.1 ; this convention agrees with the established usage unless we are working over a nonperfect base field of characteristic two. Given a one-dimensional function field $K / k$ of genus zero, our aim is to characterize intrinsically those composition algebras over $K$ which are defined over $k$.

6.2. To understand this characterization, we need to recall some facts from [P1]. Let $L$ be a field which is complete under a discrete valuation and $C$ a composition division algebra over $L$. Then the valuation of $L$ uniquely extends to a valuation of $C$, so the standard vocabulary of valuation theory applies to $C$. Setting $r=\operatorname{dim} C$, the residue class algebra $\bar{C}$ of $C$ over the residue class field $\bar{L}$ of $L$ has dimension $r$ or $\frac{r}{2}$, and is either a composition algebra or a purely inseparable field extension of characteristic two and exponent one. $C$ is said to be unramified if $\bar{C}$ is a composition algebra having $\operatorname{dim} \bar{C}=r$; otherwise, deviating from the terminology of [P1], it is said to be ramified. Finally, we call $C$ separably ramified if $\bar{C}$ is a composition algebra of dimension $\frac{r}{2}$. In this case, $C$ has the form $\operatorname{Cay}(D, \pi)$, where $D$ is an unramified composition division algebra of dimension $\frac{r}{2}$ over $L$ and $\pi$ is a prime element of the valuation ring $R$ of $L$. Writing $R_{C}, R_{D}$ for the valuation rings of $C, D$, respectively, and $\check{M}$ for the $t$-dual $\left(t=t_{C}\right)$ of an $R$-lattice $M \subset C$, it follows easily that

$$
R_{C}=R_{D} \oplus R_{D}, \quad \check{R}_{C}=R_{D} \oplus\left(\pi^{-1} R_{D}\right),
$$

so $R_{C} \subset \check{R}_{C}$ and

$$
\operatorname{dim}_{\bar{L}} \check{R}_{C} / R_{C}=r / 2 \text {. }
$$

By contrast, if $C$ is unramified, $R_{C}$ is selfdual relative to $t: \check{R}_{C}=R_{C}$.

6.3. Returning to our one-dimensional function field $K / k$ of genus zero as in 6.1 , we let $X$ be its associated nonsingular model. For any point $P \in X, \mathscr{O}_{P, X}$ is a discrete valuation ring, giving rise to a discrete valuation on $K$, trivial on $k$, so that we may form the corresponding completion, denoted by $\widehat{K}_{P}$. Following Küting [Kü], a composition algebra $C$ over $K$ is said to be unramified at $P$ in case $\widehat{C}_{P}=C \otimes_{K} \widehat{K}_{P}$ is either split or an unramified composition division algebra over $\widehat{K}_{P} ; C$ is said to be (separably) ramified at $P$ if $\widehat{C}_{P}$ is a division algebra having this property over $\widehat{K}_{P}$. For $C$ to be unramified at $P$ it is necessary and sufficient that $\widehat{C}_{P}$ contain a selfdual order. We are now ready to establish the characterization announced in 6.1 .

6.4 Theorem. Let $K / k$ be a one-dimensional function field of genus zero and $X$ its associated nonsingular model. Then a composition algebra over $K$ is defined over $k$ if and only if it is unramified at all points of $X$.

Proof. The condition is clearly necessary. Conversely, suppose $C$ is a composition algebra over $K$ which is unramified at all points of $X$. Then standard arguments from elementary lattice theory allow us to find a composition algebra $\mathscr{C}$ over $X$ whose stalk at the generic point is isomorphic to $C$. By the Classification Theorem 4.4 , we have the following possibilities for $\mathscr{C}$.

Case 1. $\mathscr{C}$ is defined over $k$. Then $C$ is defined over $k$.

Case 2. $\mathscr{C}$ is split. Then $C$ is split, hence trivially defined over $k$. 
Case 3. $\mathscr{C}$ is as in $4.4(\mathrm{iv})$. Since $K$ splits the quaternion algebra associated with $X, \mathscr{D}$ becomes split at the generic point, forcing $C$ to be split, hence defined over $k$. Q.E.D.

6.5 Remark. (i) The preceding argument shows that a composition algebra over $X$ whose stalk at the generic point is a division algebra must be defined over $k$. This corresponds to [H, Satz 3.5].

(ii) If $K=k(t)$ is rational over $k$, there is a remarkable improvement of 6.4 due to Küting [Kü, 4.3.5]: Assuming char $k \neq 2$, a composition algebra over $K$ is defined over $k$ if and only if it is unramified at all points of the affine line over $k$. The following example, modifying in a more general context an earlier one also due to Küting [Kü, 4.7.1], shows that the hypothesis on $k$ cannot be avoided.

6.6 Example. Let $k$ be a (nonperfect) field of characteristic 2 and $l=$ $k\left(\sqrt{a_{0}}, \sqrt{a_{1}}, \sqrt{a_{2}}\right)$ a purely inseparable field extension of exponent 1 and degree 8. Put $K=k(t)$ as in $6.5(\mathrm{ii})$, and let $E / K$ be the splitting field of the separable polynomial

$$
p=x^{2}+t^{-1} x+a_{0} \in K[x] .
$$

We view $E$ as a torus over $K$, which is easily seen to be unramified at all points $P \in \mathbf{A}_{k}^{1}$. By contrast, $\widehat{E}_{\infty}$ is a separable quadratic field extension of $\widehat{K}_{\infty}$, with residue class field isomorphic to $k\left(\sqrt{a_{0}}\right) / k$; hence $E$ ramifies at infinity. These properties carry over to the octonion algebra $C=\operatorname{Cay}\left(E ; a_{1}, a_{2}\right)$, thus showing that $C$ is unramified precisely at the finite places of $K / k$ without being defined over $k(6.4)$.

6.7 Remark. Küting's Characterization Theorem (6.5) rests on a result of Harder's [K, 13.4.3 and L, VI.3.13] according to which a nondegenerate symmetric bilinear form over the polynomial ring $k[t]$, char $k \neq 2$, can always be diagonalized (hence is defined over $k$ ). It turns out that the original proof of this result easily adapts to the setting of composition algebras to yield the following conclusion.

6.8 Theorem. Let $C$ be a composition algebra of rank $r$ over the polynomial ring $k[t]$ and assume char $k \neq 2$, or $r>2$ and $k$ is perfect. Then $C$ is defined over $k$.

Proof. By 3.6, we may assume that $C$ has no zero divisors, so $C^{\prime}=C \otimes_{k[t]} K$, $K=k(t)$, is a division algebra. Setting $X=\mathrm{A}_{k}^{1}=\operatorname{Spec} k[t]$ and $X^{\prime}=\mathbf{P}_{k}^{1}=$ $X \cup \infty$, we write $\mathscr{C}$ for the composition algebra over $X$ determined by $C$. Elementary lattice theory allows us to extend $\mathscr{C}$ to an algebra $\mathscr{C}^{\prime}$ over $X^{\prime}$ in such a way that $\mathscr{C}_{\infty}^{\prime}$ becomes a maximal $\mathscr{O}_{\infty, X^{\prime}}$-order in $C^{\prime}$. Although $\mathscr{C}^{\prime}$ may not be a composition algebra in general, the norm of $C^{\prime}$ induces quadratic form $N_{\mathscr{C}^{\prime}}: \mathscr{C}^{\prime} \rightarrow \mathscr{O}_{X^{\prime}}$ whose associated symmetric bilinear form has zero radical and so determines a short exact sequence

$$
0 \rightarrow \mathscr{\mathscr { C }}^{\prime} \rightarrow \check{\mathscr{C}}^{\prime} \rightarrow \mathscr{N}^{\prime} \rightarrow 0
$$

of $\mathscr{O}_{X^{\prime}}$-modules, where $\mathscr{N}^{\prime}$ is concentrated at infinity. Here we pass to the Euler characteristics and observe $\chi\left(\mathscr{N}^{\prime}\right)=h^{0}\left(X^{\prime}, \mathscr{N}^{\prime}\right)=m r / 2$, where $m=1$ or 0 according as $\mathscr{C}^{\prime}$ ramifies at infinity or not $(6.2)$; in the former case, it 
does so separably, by the hypotheses on $k$. We now invoke the Weak RiemannRoch Theorem, which says that $\chi\left(\mathscr{E}^{\prime}\right)=\operatorname{deg} \operatorname{det} \mathscr{E}^{\prime}+$ rank $\mathscr{E}^{\prime}$ for any locally free $\mathscr{O}_{X^{\prime}}$-module $\mathscr{E}^{\prime}$ of finite rank. Hence

$$
2 \operatorname{deg} \operatorname{det} \mathscr{C}^{\prime}+m r / 2=\chi\left(\mathscr{C}^{\prime}\right)-\chi\left(\check{\mathscr{C}}^{\prime}\right)+\chi\left(\mathcal{N}^{\prime}\right)=0,
$$

forcing

$$
h^{0}\left(X^{\prime}, \mathscr{C}^{\prime}\right) \geq \chi\left(\mathscr{C}^{\prime}\right)=\operatorname{deg} \operatorname{det} \mathscr{C}^{\prime}+r=(1-m / 4) r \geq 3 r / 4 .
$$

But $\Gamma\left(X^{\prime}, \mathscr{C}^{\prime}\right)$, being a finite-dimensional quadratic alternative $k$-subalgebra of the composition division algebra $C^{\prime}$ over $K$, has no zero divisors and so, by the hypotheses on $k$, is itself a composition algebra, of dimension at least $3 r / 4$. Since no number strictly between $r / 2$ and $r$ can be attained as the dimension of a composition algebra, this implies $\operatorname{dim} \Gamma\left(X^{\prime}, \mathscr{C}^{\prime}\right)=r$, so $\mathscr{C}^{\prime}$ is defined over $k$ (5.2). But then $\mathscr{C}=\left.\mathscr{C}^{\prime}\right|_{X}$ is defined over $k$ as well. Q.E.D.

6.9. In 6.8, the hypotheses on $k$ and $r$ cannot be avoided. On the one hand, Küting [Kü, 4.7.1] constructs a torus over $\mathbf{F}_{2}[t]$ not defined over $\mathbf{F}_{2}$. On the other hand, returning to 6.6 , the change of variables $y=t x$ leads to the polynomial

$$
q=y^{2}+y+t^{2} a_{0} \in k[t][y]
$$

and thus produces a torus $E^{\prime}$ over $k[t]$. Hence $\operatorname{Cay}\left(E^{\prime} ; a_{0}, a_{1}\right)$ is an octonion algebra over $k[t]$ not defined over $k$ since, if it were, the same would be true of the octonion algebra $\operatorname{Cay}\left(E ; a_{0}, a_{1}\right)$ over $k(t)$, which we have ruled out.

\section{REFERENCES}

[A] A. A. Albert, Quadratic forms permitting composition, Ann. of Math. (2) 43 (1942), 161-177.

[BS] F. van der Blij and T. A. Springer, The arithmetics of octaves and the group $G_{2}$, Nederl. Akad. Wetensch. Indag. Math. 21 (1959), 406-418.

[EGA] A. Grothendieck, Éléments de géométrie algébrique. II, Inst. Hautes Études Sci. Publ. Math. 8 (1961).

[H] G. Harder, Halbeinfache Gruppenschemata über vollständigen Kurven, Invent. Math. 6 (1968), 107-149.

[Ha] R. Hartshorne, Algebraic geometry, Graduate Texts in Math., vol. 52, Springer-Verlag, New York, Heidelberg and Berlin, 1977.

[K] M. Knebusch, Grothendieck- und Wittringe von nicht-ausgearteten symmetrischen Bilinearformen, Sitzungsber. Heidelb. Akad. Wiss. Math.-Natur. Kl., Springer-Verlag, Berlin, Heidelberg and New York, 1970.

[Kn] M.-A. Knus, Bundles on $\mathbf{P}^{2}$ with a quaternionic structure. Proc. Conf. Vector Bundles on Algebraic Varieties, Tata Institute of Fundamental Research, Bombay, 1984.

[Kü] B. Küting, Kompositionsalgebren über rationalen Funktionenkörpern, Dissertation, FernUniversität, Hagen, 1987.

[KS] R. Kunze and S. Scheinberg, Alternative algebras having scalar involutions, Pacific J. Math. 124 (1986), 159-173.

[L] T. Y. Lam, Serre's conjecture, Lecture Notes in Math., vol. 635, Springer-Verlag, Berlin, Heidelberg and New York, 1978.

[Le] S. Legrand, Généralisations d'un théorème sur les algèbres munies d'une forme quadratique multiplicative, C. R. Acad. Sci. Paris Ser. A 274 (1972), 231-234.

[M] K. McCrimmon, Nonassociative algebras with scalar involution, Pacific J. Math. 116 (1985), 85-109.

[Mi] J. S. Milne, Étale cohomology, Princeton Univ. Press, Princeton, N.J., 1980. 
[P1] H. P. Petersson, Composition algebras over a field with a discrete valuation, J. Algebra 29 (1974), 414-426.

[P2] - Composition algebras over the projective line, Contemp. Math., vol. 131, part 2, Amer. Math. Soc., Providence, R.I., pp. 673-677.

[S] R. D. Schafer, An introduction to nonassociative algebras, Academic Press, New York and London, 1966.

[T] A. Tillmann, Unzerlegbare Vektorbündel über algebraischen Kurven, Dissertation, FernUniversität, Hagen, 1983.

[W] E. Witt, Über ein Gegenbeispiel zum Normensatz, Math. Z. 39 (1934), 462-467.

[Z] L. Zagler, Ausgeartete, alternative, quadratische Algebren, Dissertation, Universität München, München, 1968.

[Zo] M. Zorn, Alternativkörper und quadratische Systeme, Abh. Math. Sem. Univ. Hamburg 9 (1933), 393-402.

Fachbereich Mathematix, FernUniversität-Gesamthochschule, Lützowstrasse 125 , D-5800 Hagen, BundesRepublik Deutschland

E-mail address: Holger.Petersson@FernUni-Hagen.de 